\title{
RESEARCH ON INFORMATION ACQUISITION AND ACCURACY ANALYSIS OF ANCIENT ARCHITECTURE PLAQUE WITH COMMON SMART PHONE
}

\author{
Xiao Pan ${ }^{1,2}$,Yungang $\mathrm{Hu}^{1,2 *}$, Miaole Hou ${ }^{1,2 *}$, Xin Zheng ${ }^{1,2}$ \\ ${ }^{1}$ Beijing University of Civil Engineering and Architecture, 102616 No.15Yongyuan Road, Daxing District, Beijing - (panxiao, \\ zhengxin)@stu.bucea.edu.cn; (huyungang, houmiaole)@.bucea.edu.cn \\ ${ }^{2}$ Beijing Key Laboratory For Architectural Heritage Fine Reconstruction \& Health Monitoring, 102616 No.15Yongyuan Road, \\ Daxing District, Beijing
}

Commission IV, ICWG IV/III, WG IV/4

KEY WORDS: Photogrammetry, 3D Reconstruction, Plaque, Ancient Architecture, Cultural Heritage

\begin{abstract}
:
The ancient architectural plaque is a unique cultural heritage of the Chinese traditional culture. It is called the "soul of ancient architecture" and has important historical and artistic value. Due to its small size and large quantity, the preservation status is not optimistic, and it is urgent to carry out rescue protection. It is especially necessary to complete its digital information collection first. The basic images used in photogrammetry are usually taken by professional cameras. With the continuous development of technology, the shooting ability of mobile phones is constantly increasing. The method of $3 \mathrm{D}$ reconstruction of plaque of ancient buildings by common mobile phone cameras is studied through experiments. Several common mobile phones were selected to collect images of ancient building plaques from different acquisition distances and 3D reconstruction experiments were carried out. The results of accuracy evaluation showed that the information of ancient architectural collected by common mobile phone cameras within the suitable acquisition range can meet the accuracy of 3D reconstruction. Requirements. In addition, the image control point measurement is replaced by a standard card. The experimental results show that the size of the model can be controlled by using the card constraint. The method can utilize the commonly used camera mobile phone to efficiently obtain the image of the ancient buildings distributed in various places and carry out three-dimensional reconstruction, thereby satisfying the urgent requirement of the urgent need for protection of the ancient buildings.
\end{abstract}

\section{INTRODUCTION}

Architectural heritage is an important part of cultural heritage and an important carrier of wisdom and spirit in ancient China. The plaque is an essential part of ancient Chinese architecture. It is made of wood or carved with brick and stone, hanging and inlaid in the building, and becomes an important symbol of an ancient building (Chen, 2003). The plaque is one of the media of Chinese national culture, and it is a unique cultural symbol of China ( $\mathrm{Yu}$, $013)$, with high social value, historical value and artistic value ( $\mathrm{Li}$ 2008; Liu et al., 2013; Zhang, 2013; Zhang, 2008). However, because the plaques of ancient buildings are mostly wooden materials and the volume is small, with the changes of the times, the retention of the plaques is not optimistic, so it is urgent to carry out corresponding information collection and protection and restoration. At present, with the country's emphasis on cultural relics protection and the development of science and technology, the complete digital archiving of cultural relics and permanent preservation has become an important aspect of protecting cultural heritage (Shang et al., 2005; Fan et al.,2010; Yang,2010). The United Nations Sustainable Development Goal 11.4 states that people should strengthen efforts to protect and safeguard the world's cultural and natural heritage.This raises the protection of cultural heritage to the level of all mankind, enough to see the importance of cultural heritage protection.

Ancient building tablets are small in volume and rectangular in shape. Therefore, it is more suitable for data acquisition by closerange photogrammetry. Photogrammetry is a related principle that relies on stereo vision. It mainly uses professional SLR cameras and image point measurement to take a multi-faceted photograph of the building body. Through the calculation of the same name, 3D coordinates are obtained, and then a 3D model is generated (Wang et al., 2001; Zhang et al., 2011; Miao et al., 2017; Wu et al., 2018). Ancient buildings are usually hanging on ancient buildings and are widely distributed and numerous. If professional equipment is used by professionals, it will cost a lot of manpower and resources, and the efficiency is not high, which is not compatible with the urgency of the current digital protection. At present, mobile phones are a necessity for the public, and their camera functions are increasingly powerful. Replacing professional SLR cameras with mobile phone cameras, mobilizing cultural relics and tourists, uploading the collected information to the network for unified data processing, will certainly improve the efficiency of digital information collection. To this end, this paper takes the ancient building plaque as the research object, and through simulation experiments to verify the results of three-dimensional modeling using mobile phone to take photos, can meet the requirements of digital collection, and then propose a method to complete the digital protection of ancient buildings using mobile phone camera .

The main problem with mobile phone cameras replacing professional SLR cameras is whether the images collected by mobile phone cameras can meet the needs of plaque information collection, and whether a method can be found to replace the measurement of image control points. Due to the variety of mobile phone brands, several common mobile phones were firstly selected for data collection experiment, and evaluate the accuracy of 3D models. Secondly, methods of image-free control point photogrammetry were found. 


\section{EXPERIMENTAL PREPARATION}

\subsection{Experimental mobile phone brand and model selection}

There are many brands and models of mobile phones on the market, and the market share of different mobile phones is not the same. Through research, according to market share and camera parameters, the following mobile phones were selected as experimental devices: Apple iphone7, HUAWEI Honor10, OPPO R17 and SAMSUNG Note8. The camera parameters are shown in Table 1 .

\begin{tabular}{|c|c|c|c|}
\hline Phone model & $\begin{array}{c}\text { Back } \\
\text { camera }\end{array}$ & Sensor & Apertrue \\
\hline $\begin{array}{c}\text { Apple } \\
\text { iphone7 }\end{array}$ & $\begin{array}{l}12 \text { million } \\
\text { pixels }\end{array}$ & BSI CMOS & $f / 1.8$ \\
\hline $\begin{array}{c}\text { HUAWEI } \\
\text { Honor } 10\end{array}$ & $\begin{array}{l}16 \text { million } \\
\text { pixels }+24 \\
\text { million } \\
\text { pixels }\end{array}$ & BSI CMOS & $f / 1.8$ \\
\hline OPPO R17 & $\begin{array}{l}16 \text { million } \\
\text { pixels }+5 \\
\text { million } \\
\text { pixels }\end{array}$ & CMOS & $\mathrm{f} / 1.7+\mathrm{f} / 2.4$, \\
\hline $\begin{array}{l}\text { SAMSUNG } \\
\text { Note8 }\end{array}$ & $\begin{array}{l}12 \text { million } \\
\text { pixels }+12 \\
\text { million } \\
\text { pixels }\end{array}$ & CMOS & $\mathrm{f} / 1.7+\mathrm{f} / 2.4$ \\
\hline
\end{tabular}

Table 1. Statistical table of camera parameters for different mobile phones

\subsection{Data processing software}

In data processing, several automatic 3D reconstruction software for tilt data that can automatically generate high-resolution 3D models from photos are compared. Image 3D modeling software uses image data as the data source, and automatically generates high-quality 3D models based on the basic principles of photogrammetry and multi-view 3D reconstruction technology. Through the comparison of software system, operation difficulty and fineness of results, a tilting photogrammetric data processing software with simple operation, high efficiency and good quality is finally selected, which is more suitable for the data processing of small-scale images (Li, 2019; Liu, 2017; He, 2017).

\subsection{Experimental subject}

In order to facilitate the experiment, a pair of plaques with a length and width of $125 \mathrm{~cm} * 40 \mathrm{~cm}$ was specially customized, as shown in Figure 2. Thirteen target points were set on the plaque for accuracy evaluation. In order to facilitate comparison, the same five points were selected as check points each time. Relative positioning measurements were made on the target using the Leica TS06 total station. The specific measured values are shown in Table 2, where the "*" punctuation is the calibration target point.

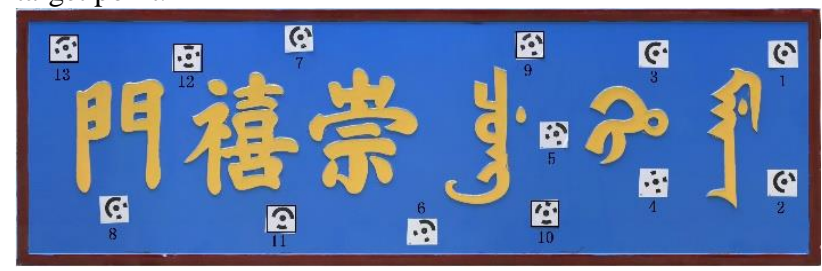

Figure 1. Target location

\begin{tabular}{|cccc|}
\hline $\begin{array}{c}\text { Point } \\
\text { number }\end{array}$ & $\mathrm{X} / \mathrm{m}$ & $\mathrm{Y} / \mathrm{m}$ & $\mathrm{Z} / \mathrm{m}$ \\
\hline 1 & 5.507 & -0.594 & -0.704 \\
2 & 5.614 & -0.597 & -0.869 \\
3 & 5.496 & -0.790 & -0.698 \\
4 & 5.609 & -0.797 & -0.863 \\
5 & 5.564 & -0.949 & -0.798 \\
6 & 5.645 & -1.159 & -0.916 \\
7 & 5.474 & -1.342 & -0.663 \\
8 & 5.615 & -1.637 & -0.877 \\
$* 9$ & 5.486 & -0.983 & -0.682 \\
$* 10$ & 5.633 & -0.969 & -0.899 \\
$* 11$ & 5.630 & -1.379 & -0.895 \\
$* 12$ & 5.488 & -1.517 & -0.684 \\
$* 13$ & 5.475 & -1.708 & -0.667 \\
\hline
\end{tabular}

Table 2. Record table of target relative coordinate

\section{EXPERIMENTAL PROCESS AND ACCURACY} ASSESSMENT

\subsection{Quotation data collection}

In data acquisition, try to make the modeled object in the format of the appropriate size. In the case where the modeled object is completely covered, the model can be completed with at least three photos, but the accuracy of the result is very low. In order to get higher quality $3 \mathrm{D}$ modeling results, you need to collect as many photos as possible. High quality imaging results and redundancy can reduce the uncertainty of matching. Therefore, in data collection, it is necessary to ensure that the horizontal and vertical overlap ratio of the image is above 50\% (Cui et al.,2016), as shown in Figure 2.

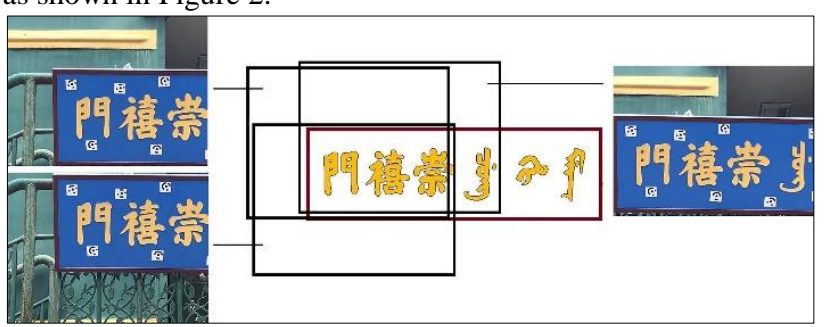

Figure 2. Schematic diagram of data acquisition

Although mobile phones are used for data acquisition, attention should also be paid to the setting requirements of shooting parameters. First of all, the lighting conditions of the shooting are very important, try to avoid overlighting scenes, and the overcast outdoor is the best shooting scene. Secondly, it is necessary to set the same photographic parameters during shooting, such as ISO, shutter, WB, etc. Adjust the ISO parameters so that the photos you get are neither too bright nor too dark. Excessive ISO settings produce more noise, which makes it difficult to match photos. The shutter needs to be adjusted slightly faster, otherwise the photo will be blurry. The camera should focus on the modeled object, otherwise it will affect the final modeling effect. One thing that needs special attention is that the Apple mobile phone needs to use the auxiliary shooting software to adjust the parameters. The camera function that comes with the Apple mobile phone cannot directly adjust the parameters. Other brands of mobile phones generally have a professional shooting mode, in which shooting parameters can be adjusted. Table 3 shows the setting of the corresponding mobile phone shooting parameters when the data is collected by the experiment. 


\begin{tabular}{|cccll|}
\hline $\begin{array}{c}\text { Phone } \\
\text { model }\end{array}$ & $\begin{array}{c}\text { Exposure } \\
\text { Time }\end{array}$ & $\begin{array}{c}\text { Sensitivi } \\
\text {-ty }\end{array}$ & $\begin{array}{c}\mathrm{F} \\
\text { Number }\end{array}$ & $\begin{array}{c}\text { Exposure } \\
\text { Mode }\end{array}$ \\
\hline $\begin{array}{c}\text { Apple } \\
\text { iphone7 }\end{array}$ & $1 / 200 "$ & ISO-20 & F1.8 & Auto \\
HUAWEI & $1 / 180 ”$ & ISO-50 & F1.8 & Auto \\
$\begin{array}{c}\text { Honor } 10 \\
\text { OPPO } \\
\text { R17 }\end{array}$ & $1 / 180 ”$ & ISO-100 & F1.7 & Auto \\
$\begin{array}{c}\text { SAMSUN } \\
\text { G Note8 }\end{array}$ & $1 / 180 ”$ & ISO-50 & F1.8 & Auto \\
\hline
\end{tabular}

Table 3. Recording table of shooting parameters setting

\subsection{Data acquisition and processing results at different distances}

At the time of data acquisition, the distance between the camera and the plaque will affect the quality of the data. In order to determine the appropriate acquisition distance, experiments need to be completed at multiple distances to make a choice. Most of the ancient buildings are hung on the door frame. For this reason, the suspension height is assumed to be about $3 \mathrm{~m}$, and the camera height is set at $1.7 \mathrm{~m}$ when the mobile phone takes photos. Under these conditions, the experimental suspension height is set to $3 \mathrm{~m}$, and the linear distance between the plaque and the camera is taken as the measured value. Five different distances including $3 \mathrm{~m}, 4 \mathrm{~m}, 5 \mathrm{~m}, 8 \mathrm{~m}$ and $10 \mathrm{~m}$ are selected to complete the data acquisition. The schematic diagram is shown in Figure 3.

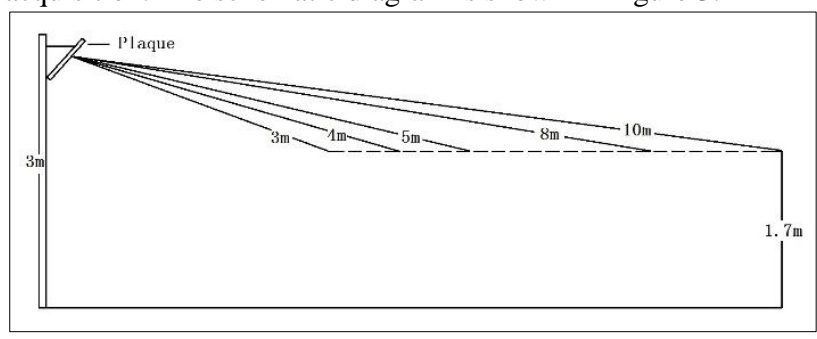

Figure 3. Schematic siagram of data acquisition

According to the requirements of the data acquisition as mentioned above, only different distances are changed, and other conditions are as consistent as possible with the parameter settings, so as to minimize accidental errors and ensure the credibility of the experiment. In addition, it ensures that the photographer is fixed and the shooting height is the same. The amount of image data for each data acquisition is approximately 25 pieces.

The collected image data is imported into the automatic 3D reconstruction software, and the previous 8 point data are input for constraint. Then the related data is processed to obtain the $3 \mathrm{D}$ model of the experimental plaque. Among them, the modeling quality parameters are set to the highest in order to obtain high quality dense point clouds and 3D models. In addition, for better comparison, the data collected by the professional SLR camera Nikon D810 is added. The lens is a zoom lens with a focal length of $70 \mathrm{~mm}$ and an ISO of 100. Figure 4 shows the orthophoto results of the experiment when the distance is chosen to be $4 \mathrm{~m}$.

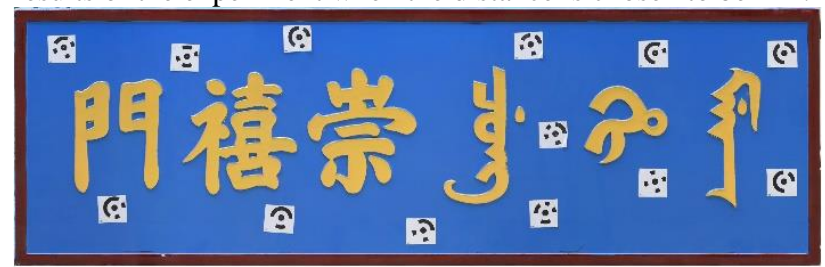

(a)

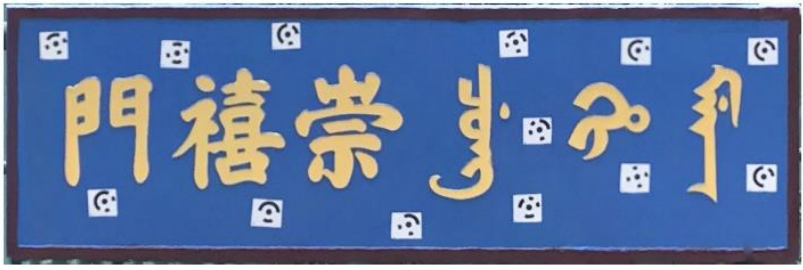

(b)

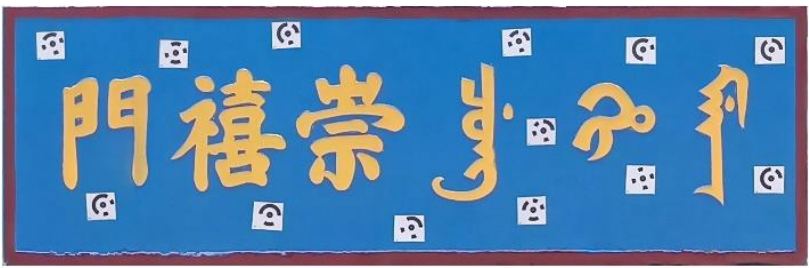

(c)

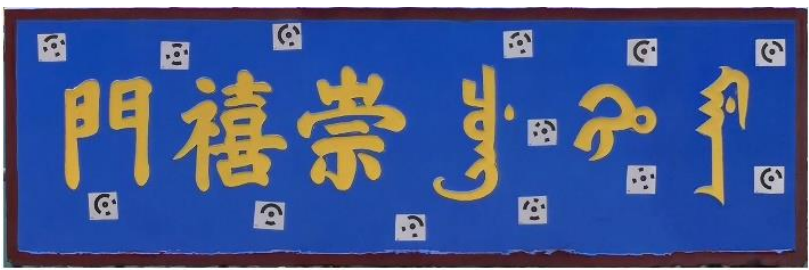

(d)

Figure 4. Orthophoto map of 3D modeling results (The shooting distance is 4 meters)

(a) Collection by Nikon D810; (b) Collection by Apple iphone7; (c) Collection by OPPO R17; (d) Collection by HUAWEI Honor10

\subsection{Accuracy evaluation of experimental results}

The accuracy evaluation of the experimental results is mainly accomplished by extracting the coordinate values of the five test points from the $3 \mathrm{D}$ model and comparing them with the corresponding coordinate values measured by the total station. When extracting the coordinate values of the checkpoint, each time the same point is extracted five times, the average value is calculated as the coordinate value of the final checkpoint. Point coordinate difference value is calculated according to formula (1) (Wu et al., 2018), and the corresponding error value is shown in Table 4.

$$
\mathrm{m}= \pm \sqrt{\Delta X^{2}+\Delta Y^{2}+\Delta Z^{2}}
$$

\begin{tabular}{|ccccccc|}
\hline Phone & Point & \multicolumn{5}{c|}{ Point coordinate difference value/mm } \\
model & num & $3 \mathrm{~m}$ & $4 \mathrm{~m}$ & $5 \mathrm{~m}$ & $8 \mathrm{~m}$ & $10 \mathrm{~m}$ \\
& -ber & & & & & \\
\hline Nikon & 9 & \pm 1.3 & \pm 1.4 & \pm 1.6 & \pm 3.1 & \pm 4.3 \\
D810 & 10 & \pm 1.3 & \pm 1.3 & \pm 1.4 & \pm 3.2 & \pm 3.7 \\
& 11 & \pm 1.7 & \pm 1.4 & \pm 1.2 & \pm 2.5 & \pm 2.6 \\
& 12 & \pm 2.2 & \pm 1.7 & \pm 1.8 & \pm 4.2 & \pm 4.3 \\
& 13 & \pm 2.0 & \pm 1.8 & \pm 2.1 & \pm 3.0 & \pm 4.2 \\
Apple & 9 & \pm 1.4 & \pm 2.0 & \pm 1.8 & \pm 15.4 & \pm 15.6 \\
iphone & 10 & \pm 1.7 & \pm 0.9 & \pm 1.9 & \pm 10.8 & \pm 13.5 \\
7 & 11 & \pm 1.0 & \pm 1.0 & \pm 1.1 & \pm 11.9 & \pm 11.0 \\
& 12 & \pm 1.8 & \pm 2.0 & \pm 2.0 & \pm 18.4 & \pm 17.6 \\
HUA & 13 & \pm 2.1 & \pm 1.9 & \pm 2.1 & \pm 20.9 & \pm 25.9 \\
WEI & 10 & \pm 1.4 & \pm 1.6 & \pm 0.8 & \pm 11.0 & \pm 13.9 \\
Honor & 11 & \pm 0.9 & \pm 0.6 & \pm 2.3 & \pm 5.7 & \pm 7.8 \\
10 & 12 & \pm 0.7 & \pm 2.1 & \pm 1.8 & \pm 8.5 & \pm 12.1 \\
& 13 & \pm 1.8 & \pm 3.3 & \pm 3.4 & \pm 16.0 & \pm 14.8
\end{tabular}




\begin{tabular}{|cclllll|} 
OPPO & 9 & \pm 1.5 & \pm 1.7 & \pm 5.0 & \pm 1.5 & \pm 18.0 \\
R17 & 10 & \pm 1.6 & \pm 1.0 & \pm 8.0 & \pm 2.6 & \pm 13.4 \\
& 11 & \pm 0.9 & \pm 0.7 & \pm 5.6 & \pm 1.2 & \pm 9.3 \\
& 12 & \pm 0.9 & \pm 2.0 & \pm 10.7 & \pm 2.3 & \pm 20.0 \\
& 13 & \pm 1.9 & \pm 2.3 & \pm 15.2 & \pm 1.9 & \pm 23.7 \\
SAMS & 9 & \pm 48.8 & \pm 10.9 & \pm 21.1 & \pm 50.6 & \pm 18.7 \\
-UNG & 10 & \pm 40.5 & \pm 11.4 & \pm 14.4 & \pm 28.4 & \pm 14.3 \\
Note8 & 11 & \pm 45.6 & \pm 5.0 & \pm 13.7 & \pm 32.3 & \pm 217.3 \\
& 12 & \pm 90.7 & \pm 13.0 & \pm 21.6 & \pm 50.1 & \pm 18.6 \\
& 13 & \pm 115.0 & \pm 29.6 & \pm 25.6 & \pm 70.4 & \pm 33.2 \\
\hline
\end{tabular}

Table 4. Statistical table of point coordinate difference value

It can be found that the data collected by the Samsung Note 8 mobile phone has a large error in the experimental results. For this reason, in the comparison and analysis of the line graph, the experimental data of Samsung Note8 is not considered. The comparison of the point coordinate difference value of the point of the experimental results of other cameras at different distances is shown in Figure 5.

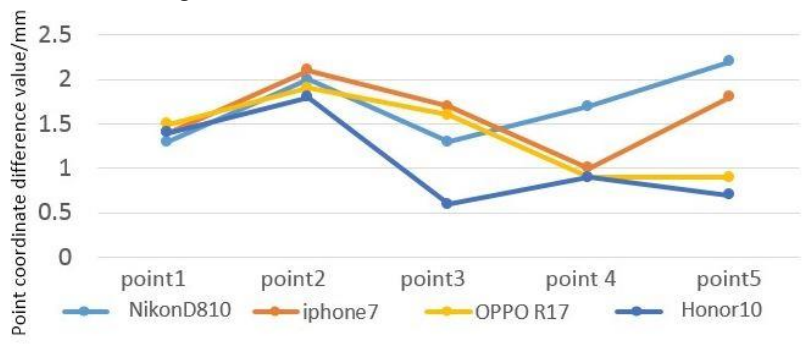

(a)

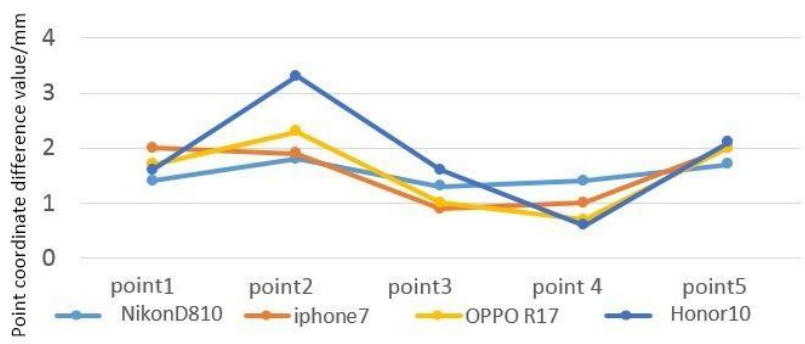

(b)

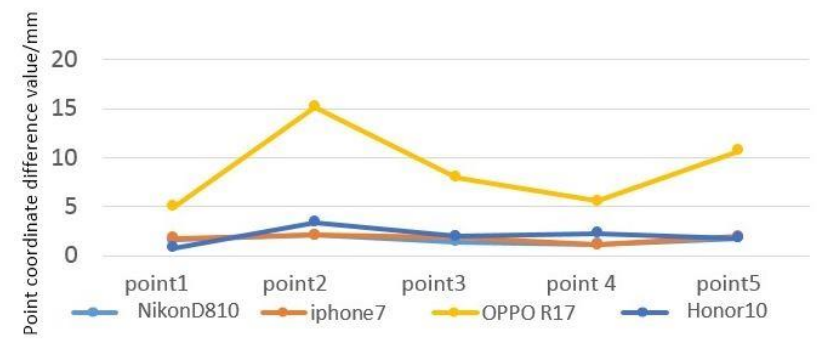

(c)

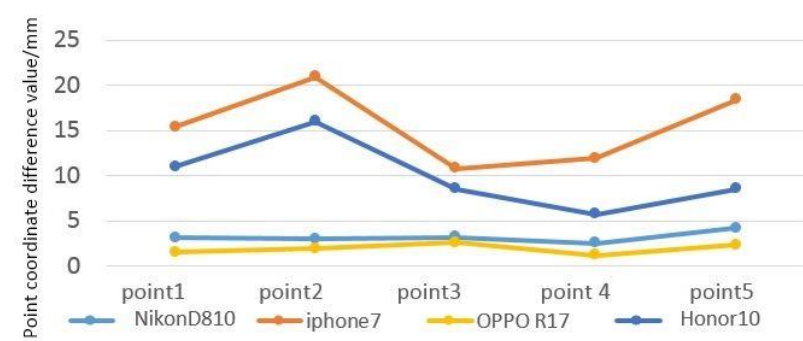

(d)

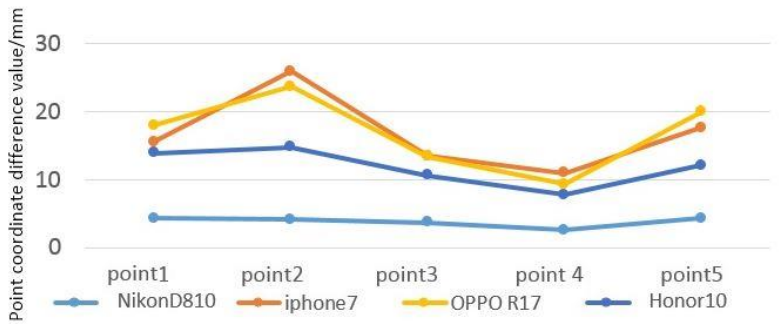

(e)

Figure 5. Comparative analysis of point coordinate difference value line chart

(a) 3 Meters distance; (b) 4 Meters distance; (c) 5 Meters distance; (d) 8 Meters distance; (e) 10 Meters distance

\subsection{Analysis of results}

Analysis of the above experimental results shows that under the same conditions, the accuracy of the 3D model obtained by the Apple iPhone7, HUAWEI Honor 10, OPPO R17 and the professional Nikon D810 SLR camera is not much different. Especially when the distance is about $4 \mathrm{~m}$, the experimental results of the mobile phone camera are almost the same as that of the professional SLR camera. The accuracy of most points is within $2 \mathrm{~mm}$, and only one point of Huawei Honor 10 has an accuracy of $3.3 \mathrm{~mm}$. For the experimental results of Samsung Note8 with a point accuracy of centimeter, the reason for the large error is that the aperture of the lens setting is large and the brightness of the image is high. This results in mismatched data processing. In order to ensure the reliability of the experiment, the collected images were not preprocessed. In the later stage, after adjusting the brightness of the image acquired by Samsung Note8, the acquisition distance is about $4 \mathrm{~m}$, and the precision is also required to reach $2 \mathrm{~mm}$.

After analysis, it can be concluded that most mobile phone cameras can meet the requirements for the collection of closerange photogrammetry data of ancient buildings under certain conditions. According to the experimental results, within a distance of about $4 \mathrm{~m}$, mobile phone camera can be used to collect data on the plaque of ancient architecture to obtain an ideal $3 d$ model with high accuracy.

\section{PROCESSING METHOD OF IMAGE-FREE CONTROL POINT MEASUREMENT}

The feasibility of using the mobile phone camera to complete the $3 \mathrm{D}$ reconstruction of the close-range photogrammetry of the ancient buildings was discussed. The total station is used for the measurement of the image control points. The starting point of this paper is to collect data by cultural enthusiasts or tourists, so it is impossible to use the total station for image point measurement. Although the 3D reconstruction can be completed only by the acquired image data, the obtained 3D model lacks absolute coordinates, and cannot extract accurate 3D coordinate values of the plaque.

For this reason, this paper considers using a regular object that people often carry in life to assist in the measurement of the image point. The selected object is a relatively common standard card, such as ID card, bank card, transportation card, etc. According to the international standard ISO7810, its length and width are unified to $85.6 \mathrm{~mm} * 53.98 \mathrm{~mm}$, as shown in Figure 6 . The specific method is to place the card near the plaque and 
collect the card and plaque on the same board when collecting data. In data processing, the length and width information of the card are used to simulate the coordinate of the image control point, and the $3 \mathrm{~d}$ reconstruction is completed jointly to obtain the $3 \mathrm{~d}$ model with real size information.

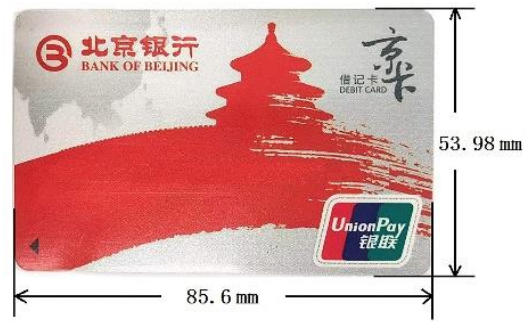

Figure 6. Identification card example diagram

In order to verify the feasibility of the method and determine its accuracy, the experiment was re-tested using the Apple iPhone $7 \mathrm{plus}$, and the data acquisition distance was chosen to be $3.5 \mathrm{~m}$, $4 \mathrm{~m}$ and $4.5 \mathrm{~m}$. In the data processing, since the cards are rounded rectangles, it is necessary to convert the rounded corners into right angles and then assign parameters before giving the card parameters. Since there are vertical relationships between any two adjacent sides of the card, two points are taken on each side of the card model, and the two points are connected as one side, and the intersection point of the side and the side is the desired right angle corner point, as shown in Figure 7. According to the length and width values of the card, the coordinate values of the four right-angle corner points are calculated as $(0,0)(0.085$ $6,0)(0.0856,0.05398)(0,0.05398)$, which can be modeled. The modeling results can be restrained again

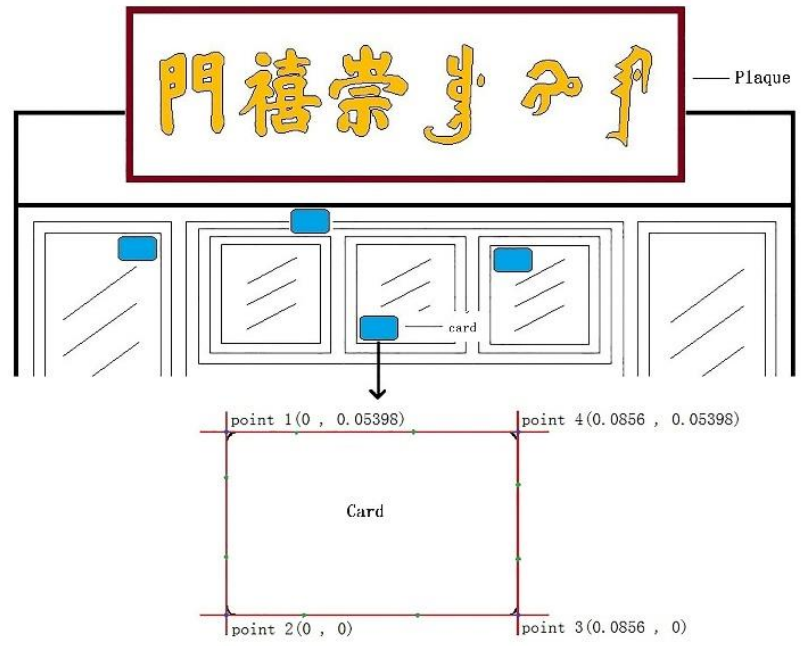

Figure 7. Card placement schematic

The coordinate values of 12 points are extracted from 13 target points on the plaque, and the accuracy was verified by comparing the calculated distance values between the points and the actual measured distance values. Under each data acquisition distance, six distances were selected (Figure 8), and each distance was measured five times in the model. The measured value is compared with the actual value on the plaque, and the root mean square error is calculated. The results are shown in Table 5. The results show that the maximum root mean square error is 0.89 $\mathrm{mm}$ and the minimum is $0.45 \mathrm{~mm}$ when the collection distance is $4 \mathrm{~m}$. The magnitude of the error is related to the position of the card, and the further the model is from the card, the greater the error. This can be done by placing the card as close as possible to the plaque to improve accuracy and ultimately achieving millimeter-level accuracy to meet data acquisition requirements.

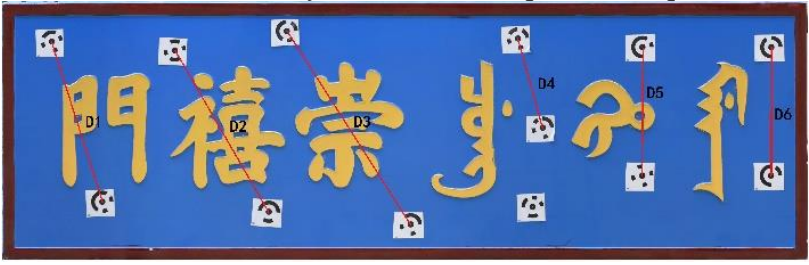

Figure 8. Line selection diagram

\begin{tabular}{|ccccccc|}
\hline Acquisition & \multicolumn{6}{c|}{ Root Mean Square Error /mm } \\
distance & D1 & D2 & D3 & D4 & D5 & D6 \\
\hline $3.5 \mathrm{~m}$ & 0.89 & 0.63 & 0.77 & 0.63 & 0.45 & 0.89 \\
$4 \mathrm{~m}$ & 0.45 & 0.77 & 0.45 & 0.89 & 0.63 & 0.77 \\
$4.5 \mathrm{~m}$ & 3.29 & 3.13 & 2.64 & 2.24 & 3.06 & 2.45 \\
\hline
\end{tabular}

Table 5. Statistical table of Root Mean Square Error

\section{PLAQUE 3D MODELING EXAMPLE}

The experimental shooting method was used to photograph the plaques of the Palace Museum. The 3D reconstruction models of the two plaques located in Yikun Palace are shown in Figure 9 and Figure 10. Through the model, it can be seen that the modeling effect is quite good, and the details such as the seal, paint stripping and ornamentation on the plaque are clear. The details of these $3 \mathrm{D}$ reconstruction models can fully meet the needs of plaque cultural relic protection, and also illustrate the feasibility of 3D reconstruction of cultural relics with the camera function of mobile phone.
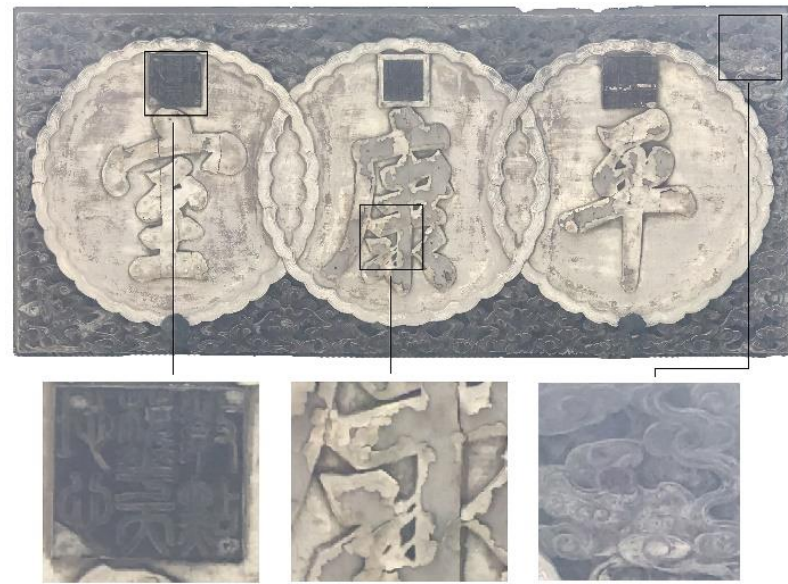

Figure 9. 3D model of the plaque "Ping Kang Shi"

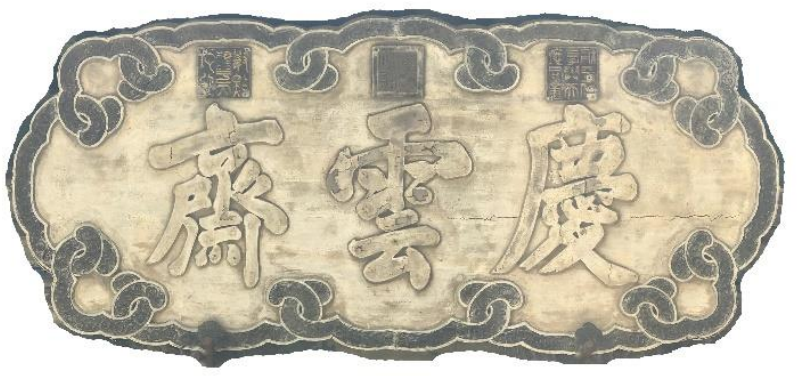

Figure 10. 3D model of the plaque "Qing Yun Zhai"

\section{CONCLUSION}

Using the mobile phone camera of cultural relics enthusiasts or tourists to collect images of ancient architectural plaques and 
upload images through the Internet, and thus efficiently complete the 3D information acquisition of the ancient buildings in various places, providing a technical idea for digital protection. By using the experimental method, 3D reconstruction experiments at different distances of selected common mobile phones are carried out. The results of precision evaluation show that the method is feasible. The method of using the standard card to replace the image point measurement is studied, so that the $3 \mathrm{D}$ reconstruction model has the correct coordinate size, and the experimental results are quite good. With the advancement of technology, the performance of mobile phone cameras is constantly improving. The use of mobile phones to record precious cultural heritage will become more and more extensive and will become a trend of digital protection of cultural heritage. This can truly make unremitting efforts for the cultural prosperity of all mankind.

\section{REFERENCES}

Chen, X. M., 2003. Plaques and couplets in Chinese architecture. Relics From South, 3, 95-96.

Yu, D., Su X. S., 2013. The plaque - a vivid wooden file of Chinese traditional culture. Lantai World, 22, 148-149.

Li, Y. H., 2008. Elementary Discussion on the Horizontal Inscribed Board Culture. Journal of Chongqing Three-Gorges University, 24 (2), 130-134.

Liu, L., Jiang, Y. Y., Liu, J. J., 2013. Discussion on the Cultural Connotation of Chinese Traditional Plaque. Art Education Research, 3, 57-57.

Zhang, Y. D., 2013. Tablet: Carriers of Intangible Culture Heritage. Academic exchanges, 4, 162-164.

Zhang, G. Q., 2014. Inscribed Boards and Couplets. Huangshan Publishing House.

Qi, H., 2014. The predicament and outlet of contemporary plaque culture. Cultural Monthly, 35, 102-105.

Shang, T., An, G. Q., 2005: Researching On the Protected Method of Ancient Building By Digital Technology. //International Conference on the Digitalization and Protection of Chinese Cultural Heritage, 147-152.

Fan, Z. W., Xing, Y., 2010. Research on the Protection of Ancient Architecture Based on Digital Technology. Beijing Surveying and Mapping, 3, 18-21.

Yang, Y., 2010. Research on Key Technologies of Digital Protection of Ancient Buildings. Henan University.

Wang, L. L., Liu, R., 2001. Review of Image-based 3D Geometric Reconstruction. Journal of System Simulation, s2, 7781 .

Zhang, R. J., Niu, M. J., Qin, N., 2011. Application of Photogrammetry in Ancient Architecture. Science \& Technology Information, 29, 74-74.

Miao, D., Wu, J., 2017. Research on 3D image reconstruction and accuracy of based on Agisoft Photoscan. Engineering of Surveying and Mapping, 26(8), 41-44.
Wu, C. Q., Zang, Z. H., Tao, X. M., 2018. Accuracy comparison of three-dimensional reconstruction between image and 3D laser scanning based on Agisoft PhotoScan. Construction Materials \& Decoration, 6, 304.

Li, H., 2019. Contrastive Analysis of Pix4Dmapper and Agistoft PhotoScan Software. Technology Wind, 20, 95.

Liu, T., 2017. Comparison of Orthophoto Processing Software for Inpho, PhotoScan and Pix4D UAVs. Technology and Industry Across the Strait, 11, 82-84.

He, Z., 2017. Comparative Study on Image Processing Software of Earthquake Emergency UAV. China Emergency Rescue, 05, $37-41$.

Cui, Z. X., Shen, Y. P., Ma, T. G., 2016. Research on Photography in Digital Three-dimensional Modeling of PhotoScan Close-range Images. Geomatics Technology and Equipment, 18(4), 10-12.

Hu, Y., 2013. Explain ISO settings in detail. DigitalCamera, 11, 80-82.

ISO/IEC 7810-2003/Amd 2-2012, Identification cards - Physical characteristics. 